\title{
Rigorous derivation of a plate theory in linear elastoplasticity via $\Gamma$-convergence
}

\author{
Matthias Liero and Thomas Roche
}

\begin{abstract}
This paper deals with dimension reduction in linearized elastoplasticity in the rate-independent case. The reference configuration of the elastoplastic body is given by a two-dimensional middle surface and a small but positive thickness. We derive a limiting model for the case in which the thickness of the plate tends to 0 . This model contains membrane and plate deformations which are coupled via plastic strains. The convergence analysis is based on an abstract $\Gamma$-convergence theory for rate-independent evolution formulated in the framework of energetic solutions. This concept is based on an energy-storage functional and a dissipation functional, such that the notion of solution is phrased in terms of a stability condition and an energy balance.
\end{abstract}

Mathematics Subject Classification (2010). 35J85, 35Q72, 49J45, 74C05, $74 \mathrm{~K} 2$.

Keywords. Linearized elastoplasticity, Rate-independent system, Hysteresis, $\Gamma$-convergence, Mosco convergence, Generalized Prandtl-Ishlinskii operator.

\section{Introduction}

For engineering applications the derivation of lower-dimensional theories for bodies such as rods, beams, membranes, plates and shells from a threedimensional theory is of fundamental importance. In $[16,17]$ a first rigorous justification of Kirchhoff's plate equation and the plane membrane system, respectively, can be found. The term "justification" has to be understood as the convergence of the solutions of the full three-dimensional system towards

Both authors wish to thank Martin Brokate, Pavel Krejčí and Alexander Mielke for insightful discussions. T. Roche thanks the Weierstrass Institute for Applied Analysis and Stochastics Berlin, where part of the work was conducted, for its kind hospitality. T. Roche was supported by the Bavarian Network of Excellence through its graduate program 'TopMath' and the TUM Graduate School through its Thematic Graduate Center 'TopMath'. 
solutions of the limiting model without any additional assumptions on the solutions. Later results for rods, linear and nonlinear plates, or shells can be found in $[3,6]$ and the references therein. An important tool in most of the recent investigations is the notion of $\Gamma$-convergence. This convergence assures, roughly speaking, that (almost) minimizers of the three-dimensional theory (subject to suitable boundary conditions and applied loads) converge to minimizers of the limiting lower-dimensional theory.

However, as $\Gamma$-convergence is a purely static concept, there are only very few results concerning the justification of similar dimension reductions for evolutionary problems in nonlinear continuum mechanics, see [1] for a recent result. More often, lower dimensional theories are derived by ad hoc assumptions via formal asymptotic expansion, see e.g. [7,9,15].

In [11] an elastoplastic plate model in the rate-independent case was derived using an abstract $\Gamma$-convergence result developed in [13]. The scaling of the displacements in [11] follows the classical theory, see e.g. [4] and the references therein. However, the plastic strains were scaled in a way such that the dissipation potential of the scaled system is independent of the parameter describing the thickness of the plate. Hence, it can be shown that the scaled dissipation functional converges continuously to a limit functional and the results of [13] can be directly applied.

In this paper we propose a scaling of the plastic strains that matches the scaling of the linearized strain tensor. Therefore, the scaled dissipation functional depends on the thickness of the plate and converges in the sense of Mosco (see [2]) to a highly degenerated limit functional. Hence the method developed in [11] cannot be applied. By exploiting the quadratic form of the energy functionals we are in position to circumvent this problem and to do a limit passage from linearized elastoplasticity in three dimensions to a model that combines two two-dimensional linear elastic models, namely the membrane model for in-plane displacements and Kirchhoff's plate equation for the out-of-plane displacement, with plastic effects. Although the equations for the elastic equilibrium are the same as in [11] the plastic flow rule differs.

The evolution of an elastoplastic body in the rate-independent case can be formulated in different ways, e.g. as a variational inequality, a differential inclusion, or as an energetic system. All three are expressed in terms of an energy functional

$$
\mathcal{E}^{h}(t, u, p)=\int_{\Omega} W^{h}(\varepsilon(u), p) \mathrm{d} x-\langle\ell(t), u\rangle,
$$

defined as integral over the rescaled plate domain $\Omega:=\omega \times(-1 / 2,1 / 2)$. Here $u$ and $p$ are the rescaled displacements and plastic strains, respectively. The small parameter $h>0$ is proportional to the unscaled thickness of the plate and occurs in $W_{h}$ via the corresponding scalings of the strains.

Additionally we have a dissipation potential

$$
\mathcal{R}^{h}(\dot{p})=\int_{\Omega} R^{h}(\dot{p}) \mathrm{d} x .
$$


Rate-independence is implemented by the positive 1-homogeneity of $R^{h}$, i.e. $R^{h}(\lambda \dot{p})=\lambda R^{h}(\dot{p})$ for all $\lambda \geq 0$ and all $\dot{p}$.

The solutions have to satisfy the differential inclusion

$$
0=\mathrm{D} \mathcal{E}^{h}(t, u(t), p(t)), \quad 0 \in \partial_{\dot{p}} \mathcal{R}^{h}(\dot{p}(t))+\mathrm{D}_{p} \mathcal{E}^{h}(t, u(t), p(t)),
$$

where the first equation is the balance of forces and the second is the plastic flow rule.

For a quadratic energy functional $\mathcal{E}^{h}(t, \cdot)$ the differential inclusion is fully equivalent to the so-called energetic formulation (see $[12,14])$. The energetic formulation is stated in terms of an energetic stability condition and the total balance of energy. The advantage of the energetic formulation is that it is based on $\mathcal{E}^{h}$ and $\mathcal{R}^{h}$ rather than on their derivatives. Thus, notions of convergence for functionals such as $\Gamma$-convergence and Mosco convergence can be applied.

The underlying model together with the underlying scalings will be described in Sect. 2.1. Moreover, we state the main result of this paper, namely the convergence of the solutions of the three-dimensional system to a solution of a lower-dimensional system. Its proof is the content of the following Sect. 3. Here, we use the ideas developed in [13].

In Sect. 4 we formulate the limit problem in terms of the in-plane displacements $\left(v_{1}, v_{2}\right)$, the out-of-plane displacement $v_{3}$, and the plastic strain $p$, which is still defined on all of $\Omega$. For an isotropic material, the limiting model takes the form

$$
\begin{aligned}
& \left.0=-\operatorname{div}\left(\Sigma_{0}\left(\varepsilon^{1,2}(v)\right)-\left[p^{1,2}\right]_{0}\right)\right)-G_{\text {memb }}(t, \cdot) \quad \text { in } \omega \\
& 0=\operatorname{div} \operatorname{div}\left(\Sigma_{0}\left(\frac{1}{24} \mathrm{D}^{2} v_{3}+\left[p^{1,2}\right]_{1}\right)\right)-g_{\text {bend }}(t, \cdot)-\operatorname{div} G_{\text {bend }}(t, \cdot) \quad \text { in } \omega \\
& 0 \in \partial R(\mathbb{D} \dot{p})+\left(\llbracket \Sigma_{0}\left(p^{1,2}-\varepsilon^{1,2}(v)+x_{3} \mathrm{D}^{2} v_{3}\right) \| 0 \rrbracket\right)+k_{\text {hard }} \mathbb{D} p \quad \text { in } \Omega
\end{aligned}
$$

where $\Sigma_{0}(\varepsilon):=\frac{2 \lambda \mu}{\lambda+2 \mu} \operatorname{tr} \varepsilon I_{2}+2 \mu \varepsilon, \varepsilon \in \mathbb{R}_{\text {sym }}^{2 \times 2}$. Here, $\varepsilon^{1,2}(v) \in \mathbb{R}_{\text {sym }}^{2 \times 2}$ is the in-plane strain tensor and $\mathrm{D}^{2} v_{3} \in \mathbb{R}_{\text {sym }}^{2 \times 2}$ the bending strain tensor.

Equation (1.1a) is the second-order membrane equation for $\left(v_{1}, v_{2}\right)$, which is coupled to the plastic strain $p$ via the integrals $[\cdot]_{0}$ over $x_{3} \in(-1 / 2,1 / 2)$. Equation (1.1b) is a generalization of Kirchhoff's plate equation (of order four) for $v_{3}$. It is also coupled to the plastic strain $p$, but now with weighted averages $[\cdot]_{1}$. The flowrule $(1.1 \mathrm{c})$ exhibits the elastic strains as forcing in a very special manner concerning the dependence on $x_{3}$.

In Sect. 4.1 we discuss other possible choices for the scalings of the plastic strain and compare the results with the limit model derived in [11]. Finally, in Sect. 4.2 we show briefly how the last equation in (1.1) can be eliminated using a vector-valued hysteresis operator of play type. 


\section{Setup of the elastoplastic model}

The starting point for our study is the classical elastoplastic model with hardening. Here we focus on domains with plate geometry, i.e., $\Omega_{h}=\omega \times$ $(-h / 2, h / 2)$, where $\omega$ is the mid surface and the thickness $h>0$ is sufficiently small. We formulate the evolution of the plate in terms of a differential inclusion or equivalently as a variational inequality. Moreover, we will outline the suitable scalings to obtain a nontrivial limiting model. The final model will be presented in Sect. 2.3, while the convergence proof is the content of Sect. 3.

\subsection{The clamped elastoplastic plate}

We consider a bounded Lipschitz domain $\omega \subset \mathbb{R}^{2}$ and set $\Omega_{h}:=\omega \times$ $(-h / 2, h / 2)$. We denote by $\Gamma_{h}^{0}=\gamma_{0} \times(-h / 2, h / 2)$ the part of the body with prescribed boundary conditions. Here, $\gamma_{0} \subset \partial \omega$ has a positive 1-dimensional Hausdorff measure. We set

$$
\mathrm{H}_{\Gamma_{h}^{0}}^{1}\left(\Omega_{h} ; \mathbb{R}^{3}\right):=\left\{u \in \mathrm{H}^{1}\left(\Omega_{h} ; \mathbb{R}^{3}\right): u=0 \text { on } \Gamma_{h}^{0}\right\},
$$

where $\Gamma_{h}^{0}:=\gamma_{0} \times(-h / 2, h / 2)$ denotes the part of the boundary where the displacement is prescribed.

The elastoplastic properties of the body $\Omega_{h}$ are described in terms of the linearized strain tensor $\varepsilon(u)=\frac{1}{2}\left(\nabla u+\nabla u^{\top}\right)$ and the plastic strain tensor

$$
p \in \mathbb{R}_{\mathrm{dev}}^{3 \times 3}:=\left\{A \in \mathbb{R}_{\mathrm{sym}}^{3 \times 3}: \operatorname{tr} A=0\right\}
$$

via the stored energy density $\mathbb{W}: \mathbb{R}_{\mathrm{sym}}^{3 \times 3} \times \mathbb{R}_{\mathrm{dev}}^{3 \times 3} \rightarrow \mathbb{R}$, which is assumed to be given by

$$
\mathbb{W}(\varepsilon, p)=\frac{1}{2} \mathcal{C}(\varepsilon-p):(\varepsilon-p)+\frac{k_{\text {hard }}}{2}|p|^{2},
$$

where ':' denotes the usual inner product on $\mathbb{R}^{3 \times 3}$, i.e. $A: B=\operatorname{tr}\left(A^{T} B\right)$. Here we are interested in the isotropic and homogeneous case, i.e., $\mathcal{C}$ e $=\lambda \operatorname{tr} \mathrm{e}+2 \mu \mathrm{e}$, where $\lambda, \mu>0$ are the Lamé constants and $k_{\text {hard }}$ is a measure for kinematic hardening.

Moreover, the plastic flow rule of the material can be formulated in terms of a dissipation potential $R: \mathbb{R}_{\mathrm{dev}}^{3 \times 3} \rightarrow[0, \infty)$, which is assumed to be continuous, convex, and homogeneous of degree 1. The latter conditions means $R(\lambda \dot{p})=\lambda R(\dot{p})$ for all $\lambda \geq 0$ and $\dot{p} \in \mathbb{R}_{\mathrm{dev}}^{3 \times 3}$. The corresponding elastic domain $K \subset \mathbb{R}_{\mathrm{dev}}^{3 \times 3}$ is defined via $K:=\partial R(0)$, which is the subdifferential of $R$ at 0 . More specifically, we assume that $R(\dot{p})=\sigma_{\text {yield }}|\dot{p}|$ for a given yield stress $\sigma_{\text {yield }}>0$. This corresponds to the von Mises yield criterion.

Given time-dependent volume and surface loadings $f_{h}(t, \cdot)$ and $g_{h}(t, \cdot)$ the full elastoplastic problem can be written in the form

$$
\begin{array}{ll}
-\operatorname{div}\left(\partial_{\varepsilon} \mathbb{W}(\varepsilon(u), p)\right)=f_{h}(t, \cdot) & \text { in } \Omega_{h}, \\
0 \in \partial R(\dot{p})+\partial_{p} \mathbb{W}(\varepsilon(u), p) & \text { in } \Omega_{h}, \\
u(t, \cdot)=0 & \text { on } \Gamma_{h}^{0}, \\
\partial_{\varepsilon} \mathbb{W}(\varepsilon(u), p) \nu=g_{h}(t, \cdot) & \text { on } \partial \Omega_{h} \backslash \Gamma_{h}^{0},
\end{array}
$$


where $\nu$ denotes the outer normal vector on $\partial \Omega_{h}$. Here $\sigma=\partial_{\varepsilon} \mathbb{W} \in \mathbb{R}_{\text {sym }}^{3 \times 3}$ denotes the stress, while $\partial_{p} \mathbb{W} \in \mathbb{R}_{\text {dev }}^{3 \times 3}$ contains the deviator of the stress as well as any plastic back stresses.

We reformulate the system (2.1) in abstract form for the pair $q=(u, p)$ via the energy functional $\mathcal{E}_{h}:[0, T] \times \mathcal{Q}_{h} \rightarrow \mathbb{R}$ and the dissipation functional $\mathcal{R}_{h}: \mathcal{Q}_{h} \rightarrow[0, \infty]$ as follows

$$
\begin{gathered}
\mathcal{Q}_{h}:=\mathrm{H}_{\Gamma_{h}^{0}}^{1}\left(\Omega_{h} ; \mathbb{R}^{3}\right) \times \mathrm{L}^{2}\left(\Omega_{h} ; \mathbb{R}_{\mathrm{dev}}^{3 \times 3}\right) \\
\mathcal{E}_{h}(t, q):=\int_{\Omega_{h}} \mathbb{W}(\varepsilon(u), p) \mathrm{d} x-\left\langle\ell_{h}(t), u\right\rangle \text { and } \mathcal{R}_{h}(\dot{p}):=\int_{\Omega_{h}} R(\dot{p}) \mathrm{d} x .
\end{gathered}
$$

where $\ell_{h}(t) \in \mathcal{Q}_{h}^{*}$ is defined via

$$
\left\langle\ell_{h}(t), u\right\rangle:=\int_{\Omega} f_{h}(t, x) \cdot u(x) \mathrm{d} x+\int_{\bar{\Omega}_{h} \backslash \Gamma_{h}^{0}} g_{h}(t, x) \cdot u(x) \mathrm{d} a(x) .
$$

Although the dissipation potential depends only on the plastic strain rate $\dot{p}$ we will also write $\mathcal{R}_{h}(\dot{q})$ as no confusion will arise.

We call a function $q_{h}=\left(u_{h}, p_{h}\right):[0, T] \rightarrow \mathcal{Q}_{h}$ a solution to the RIS $\left(\mathcal{Q}_{h}, \mathcal{E}_{h}, \mathcal{R}_{h}\right)$ [and hence to the above elastoplastic problem (2.1)], if it solves one of the following three equivalent problem formulations:

Differential inclusion:

$$
0 \in \partial \mathcal{R}_{h}\left(\dot{q}_{h}(t)\right)+\mathrm{D}_{q} \mathcal{E}_{h}\left(t, q_{h}(t)\right)
$$

Variational inequality:

$$
\forall \widetilde{q} \in \mathcal{Q}_{h}:\left\langle\mathrm{D}_{q} \mathcal{E}_{h}\left(t, q_{h}(t)\right), \widetilde{q}-\dot{q}\right\rangle+\mathcal{R}_{h}(\widetilde{q})-\mathcal{R}_{h}\left(\dot{q}_{h}\right) \geq 0 ;
$$

Energetic formulation:

(S) $\forall \widetilde{q} \in \mathcal{Q}_{h}: \mathcal{E}_{h}\left(t, q_{h}(t)\right) \leq \mathcal{E}_{h}(t, \widetilde{q})+\mathcal{R}_{h}\left(\widetilde{q}-q_{h}(t)\right)$,

(E) $\mathcal{E}_{h}(t, q(t))+\int_{0}^{t} \mathcal{R}_{h}(\dot{q}) \mathrm{d} s=\mathcal{E}_{h}\left(0, q_{h}(0)\right)-\int_{0}^{t}\left\langle\dot{\ell}_{h}, q_{h}\right\rangle \mathrm{d} s$.

Condition $(\mathrm{S})$ is referred to as the stability whereas $(\mathrm{E})$ is called the energy balance. We refer to [12, Sect.2] for the equivalence between these three forms. For any $h>0$ and $\ell_{h} \in \mathrm{W}^{1,1}\left(0, T ; \mathcal{Q}_{h}^{*}\right)$ the above problems have a unique solution (see $[8,14]$ )

\subsection{Scaling for thin-plate domains}

It is already known from the theory of linearized elasticity, see $[3,16,17]$ that the strain of in-plane displacements (membrane modes) are smaller than the out-of-plane modes (bending modes). As in [11] we use the following scaling for the displacements:

$$
u_{h}\left(x_{h}\right)=S_{h} u^{h}\left(S_{h} x_{h}\right), \quad \text { where } S_{h}=\operatorname{diag}(1,1,1 / h) .
$$

Since $x_{h} \in \Omega_{h}$ is mapped to $x=S_{h} x_{h} \in \Omega_{1}$, the rescaled function $u^{h}$ will be defined in $\mathcal{U}:=\mathrm{H}_{\Gamma^{0}}^{1}\left(\Omega ; \mathbb{R}^{3}\right)$, where $\Omega:=\Omega_{1}$ and $\Gamma^{0}:=\gamma_{0} \times(-1 / 2,1 / 2)$. In the following we will indicate functions, functionals etc. associated with the domain $\Omega_{h}$ by a subscript $h$ and their rescaled counterparts by a superscript $h$. 
For linearized elasticity the scaling of the strains is arbitrary, because it is an infinitesimal theory by definition. In contrast, the theory of linearized elastoplasticity is no longer scaling invariant, because the boundary of the elastic domain $K=\partial R(0)$ contains the given yield stresses of order 1, i.e. independent of $h$. Thus, our theory needs a scaling where most of the strains in the plastic tensor $p$ as well as in $\varepsilon$ are of order 1 .

The scaling acts differently on the components of the strains in $\varepsilon\left(u_{h}\right)$, as follows

$$
\varepsilon\left(u_{h}\right)\left(x_{h}\right)=S_{h} \varepsilon\left(u^{h}\right)\left(S_{h} x_{h}\right) S_{h}=\left(\begin{array}{lll}
\varepsilon_{11}\left(u^{h}\right) & \varepsilon_{12}\left(u^{h}\right) & \frac{1}{h} \varepsilon_{12}\left(u^{h}\right) \\
\varepsilon_{12}\left(u^{h}\right) & \varepsilon_{22}\left(u^{h}\right) & \frac{1}{h} \varepsilon_{23}\left(u^{h}\right) \\
\frac{1}{h} \varepsilon_{13}\left(u^{h}\right) & \frac{1}{h} \varepsilon_{23}\left(u^{h}\right) & \frac{1}{h^{2}} \varepsilon_{33}\left(u^{h}\right)
\end{array}\right) .
$$

Concerning the scaling of the plastic strain tensor we look for scalings of the form

$$
p_{h}\left(x_{h}\right)=\Pi_{h}^{\alpha, \beta} p^{h}\left(S_{h} x_{h}\right):=\left(\begin{array}{lll}
p_{11}^{h} & p_{12}^{h} & \frac{1}{h^{\alpha}} p_{13}^{h} \\
p_{12}^{h} & p_{22}^{h} & \frac{1}{h^{\alpha}} p_{23}^{h} \\
\frac{1}{h^{\alpha}} p_{13}^{h} & \frac{1}{h^{\alpha}} p_{23}^{h} & \frac{1}{h^{\beta}} p_{33}^{h}
\end{array}\right) .
$$

To simplify the presentation we will choose $\alpha=1$ and $\beta=2$ which fits to the scaling of $\varepsilon$, i.e, $p_{h}=S_{h} p^{h} S_{h}$. Note, that this differs from the scalings in [11] where $\alpha=\beta=0$. We refer to the end of Sect. 4 for a discussion of more general scalings. The plastic strain tensors $p^{h}$ are thus defined in the space

$$
\mathcal{P}^{h}:=\left\{p \in \mathrm{L}^{2}\left(\Omega ; \mathbb{R}_{\mathrm{sym}}^{3 \times 3}\right): p_{11}+p_{22}+p_{33} / h^{2}=0\right\} \subset \mathcal{P}:=\mathrm{L}^{2}\left(\Omega ; \mathbb{R}_{\mathrm{sym}}^{3 \times 3}\right) .
$$

Finally, we introduce the spaces

$$
\mathcal{Q}^{h}:=\mathcal{U} \times \mathcal{P}^{h} \subset \mathcal{Q}:=\mathcal{U} \times \mathcal{P} .
$$

When substituting $q^{h}=\left(u^{h}, p^{h}\right)$ in $\mathcal{E}^{h}$ and $\mathcal{R}^{h}$ we still have to take care of the change in the volume measure. Hence we set

$$
\mathcal{E}^{h}\left(t, u^{h}, p^{h}\right)=\frac{1}{h} \mathcal{E}_{h}\left(t, u_{h}, p_{h}\right) \quad \text { and } \quad \mathcal{R}^{h}\left(\dot{p}^{h}\right)=\frac{1}{h} \mathcal{R}_{h}\left(\dot{p}_{h}\right)
$$

To control the loading part of $\ell_{h}$ defined in (2.2), we also have to assume a corresponding scaling of the loadings namely

$$
f_{h}\left(t, x_{h}\right)=S_{h}^{-1} F_{\mathrm{vol}}\left(t, S_{h} x_{h}\right) \text { and } g_{h}\left(t, x_{h}\right)=h S_{h}^{-1} F_{\mathrm{surf}}\left(t, S_{h} x_{h}\right),
$$

where $x_{h} \in \omega \times\{-h / 2, h / 2\}$. For simplicity, we assume that there are no surface loadings on $\partial \omega \backslash \gamma_{0} \times(-h / 2, h / 2)$. They could be easily included, but need a different scaling. Then, $\mathcal{E}^{h}:[0, T] \times \mathcal{Q}^{h} \rightarrow \mathbb{R}$ and $\mathcal{R}^{h}: \mathcal{Q}_{h} \rightarrow[0, \infty)$ take the form

$$
\begin{aligned}
\mathcal{E}^{h}(t, u, p) & =\int_{\Omega} \mathbb{W}\left(S_{h} \varepsilon(u) S_{h}, S_{h} p S_{h}\right) \mathrm{d} x-\langle\ell(t), u\rangle, \\
\mathcal{R}^{h}(\dot{p}) & =\int_{\Omega} R\left(S_{h} \dot{p}(x) S_{h}\right) \mathrm{d} x, \\
\langle\ell(t), u\rangle & =\int_{\Omega} F_{\mathrm{vol}}(t, x) u(x) \mathrm{d} x+\int_{\omega \times\{-1,1\}} F_{\text {surf }}(t, x) u(x) \mathrm{d} a(x),
\end{aligned}
$$


where $F_{\text {vol }}$ and $F_{\text {surf }}$ are such that $\ell \in \mathrm{W}^{1,1}\left(0, T ; \mathcal{U}^{*}\right)$. In order to compute the $\Gamma$-limits for $\mathcal{E}^{h}$ and $\mathcal{R}^{h}$, we extend $\mathcal{E}^{h}$ and $\mathcal{R}^{h}$ to the bigger space $\mathcal{Q}$ by setting $\mathcal{E}^{h}=\mathcal{R}^{h}=\infty$ on $\mathcal{Q} \backslash \mathcal{Q}^{h}$.

The only dependence in $h$ occurs through the scaling of the elastic and plastic strains. Using Korn's inequality and assuming $h \in(0,1]$ we have the uniform convexity.

$$
\begin{aligned}
\mathcal{E}^{h}(t, q) & \geq c\left(\left\|S_{h} \varepsilon(u) S_{h}\right\|_{\mathrm{L}^{2}}^{2}+\left\|S_{h} p S_{h}\right\|_{\mathrm{L}^{2}}^{2}\right) \geq c\left(\|\varepsilon(u)\|_{\mathrm{L}^{2}}^{2}+\|p\|_{\mathrm{L}^{2}}^{2}\right) \\
& \geq c_{\mathrm{Korn}} c\|u\|_{\mathrm{H}^{1}}^{2}+c\|p\|_{\mathrm{L}^{2}}^{2}
\end{aligned}
$$

independently of $h$. The existence of solutions of the RIS $\left(\mathcal{Q}, \mathcal{E}^{h}, \mathcal{R}^{h}\right)$ is classical. For a proof of the following theorem we refer to $[8,14,18]$.

Theorem 2.1. Assume that $\left(\mathcal{Q}, \mathcal{E}^{h}, \mathcal{R}^{h}\right)$ is as above with $\ell \in \mathrm{W}^{1,1}\left(0, T ; \mathcal{Q}^{*}\right)$ and that $q_{0}^{h} \in \mathcal{Q}$ is stable at $t=0$ (i.e. $0 \in \partial \mathcal{R}^{h}(0)+\mathrm{D}_{q} \mathcal{E}^{h}\left(0, q_{0}^{h}\right)$ ), then there is a unique energetic solution $q^{h} \in \mathrm{W}^{1,1}(0, T ; \mathcal{Q})$ with $q^{h}(0)=q_{0}^{h}$. Moreover, it holds that $q^{h}(t) \in \mathcal{Q}^{h}$ and

$$
\left\|\dot{q}^{h}\right\|_{\mathrm{L}^{1}(r, s ; \mathcal{Q})} \leq C\|\dot{\ell}(t)\|_{\mathrm{L}^{1}\left(r, s ; \mathcal{Q}^{*}\right)},
$$

where $C>0$ is independent of $h$ and $0 \leq r<s \leq T$.

Obviously, the scalings of the unique energetic solutions w.r.t. the RIS (rate-independent system) $\left(\mathcal{Q}_{h}, \mathcal{E}_{h}, \mathcal{R}_{h}\right)$ are the unique energetic solutions of the RIS $\left(\mathcal{Q}^{h}, \mathcal{E}^{h}, \mathcal{R}^{h}\right)$.

\subsection{The limiting elastoplastic model}

Obviously, the energy $\mathcal{E}^{h}$ blows up for $h \rightarrow 0$ if the strains $\varepsilon_{i 3}(u)$ and $p_{i 3}$ do not vanish. Thus, we expect the limit energy to be defined on a reduced space, namely

$$
\mathcal{Q}_{\mathrm{KL}}:=\left\{(u, p) \in \mathcal{Q}: \varepsilon_{i 3}(u)=p_{i 3}=0\right\}=\mathcal{U}_{\mathrm{KL}} \times \mathcal{P}_{\mathrm{KL}} .
$$

The restriction in $\mathcal{U}_{\mathrm{KL}}$ take the explicit form

$$
\partial_{x_{1}} u_{3}+\partial_{x_{3}} u_{1}=\partial_{x_{2}} u_{3}+\partial_{x_{3}} u_{2}=\partial_{x_{3}} u_{3}=0 \text { a.e. in } \Omega \text {. }
$$

The last equation implies that $u_{3}$ is independent of $x_{3}$. Using this the first two equations imply that $u_{1}$ and $u_{2}$ are affine in $x_{3}$. Defining

$$
V=\left\{\left(v_{1}, v_{2}, v_{3}\right) \in \mathrm{H}_{\gamma_{0}}^{1}\left(\omega ; \mathbb{R}^{3}\right): v_{3} \in \mathrm{H}^{2}(\omega), \nabla v_{3} \cdot \nu=0 \text { on } \gamma_{0}\right\}
$$

the space $\mathcal{U}_{\mathrm{KL}}$ of so-called Kirchhoff-Love displacements can be characterized by

$$
\begin{aligned}
& \mathcal{U}_{\mathrm{KL}}=\{u=\mathcal{K} v: v \in V\} \text { with } \\
& \mathcal{K} v\left(x_{1}, x_{2}, x_{3}\right)=\left(\begin{array}{c}
v_{1}\left(x_{1}, x_{2}\right)-x_{3} \partial_{x_{1}} v_{3}\left(x_{1}, x_{2}\right) \\
v_{2}\left(x_{1}, x_{2}\right)-x_{3} \partial_{x_{2}} v_{3}\left(x_{1}, x_{2}\right) \\
v_{3}\left(x_{1}, x_{2}\right)
\end{array}\right),
\end{aligned}
$$

see e.g. $[3,4]$. Note that the component $u_{3}$ has gained higher smoothness.

The limit model will be defined in such a way that it is restricted to $\mathcal{U}_{\mathrm{KL}} \times \mathcal{P}_{\mathrm{KL}}$. The reduced energy is obtained by relaxing the strains $\varepsilon_{j 3}$ and $p_{j 3}$ 
in the following way. We decompose the 6 -dimensional space $\mathbb{R}_{\text {sym }}^{3 \times 3}$ into two three-dimensional components by setting

$$
\varepsilon^{1,2}:=\left(\begin{array}{ll}
\varepsilon_{11} & \varepsilon_{12} \\
\varepsilon_{21} & \varepsilon_{22}
\end{array}\right) \in \mathbb{R}_{\mathrm{sym}}^{2 \times 2}, \quad \varepsilon^{3}:=\left(\varepsilon_{13}, \varepsilon_{23}, \varepsilon_{33}\right) \in \mathbb{R}^{3} .
$$

For $A \in \mathbb{R}_{\mathrm{sym}}^{2 \times 2}$ and $b \in \mathbb{R}^{3}$ we define $\llbracket A \| b \rrbracket \in \mathbb{R}_{\mathrm{sym}}^{3 \times 3}$ such that $\varepsilon=\llbracket \varepsilon^{1,2} \| \varepsilon^{3} \rrbracket$, i.e.

$$
\llbracket A \| b \rrbracket=\left(\begin{array}{lll}
A_{11} & A_{12} & b_{1} \\
A_{12} & A_{22} & b_{2} \\
b_{1} & b_{2} & b_{3}
\end{array}\right)
$$

Now we define a relaxed energy density depending only on $\varepsilon^{1,2}$ and $p^{1,2}$, namely $\overline{\mathbb{W}}\left(\varepsilon^{1,2}, p^{1,2}\right):=\min \left\{\mathbb{W}\left(\llbracket \varepsilon^{1,2}\left\|a \rrbracket, \llbracket p^{1,2}\right\| b \rrbracket\right): a, b \in \mathbb{R}^{3} \wedge b_{3}=-p_{11}-p_{22}\right\}$.

Note that due to the plastic incompressibility the constraint $b_{3}=-p_{11}-p_{22}$ has to be included. The definition of $\overline{\mathbb{W}}$ implies the important lower estimate

$$
\mathbb{W}\left(S_{h} \varepsilon S_{h}, S_{h} p S_{h}\right) \geq \overline{\mathbb{W}}\left(\varepsilon^{1,2}, p^{1,2}\right) \quad \text { for all } h \in[0,1], \varepsilon, p \in \mathbb{R}_{\mathrm{sym}}^{3 \times 3} .
$$

For the isotropic $\mathbb{W}$ defined in Sect. 2.1 we obtain the energy density

$$
\begin{aligned}
& \overline{\mathbb{W}}\left(\varepsilon^{1,2}, p^{1,2}\right) \\
& =\frac{\lambda \mu}{\lambda+2 \mu}\left(\operatorname{tr}\left(\varepsilon^{1,2}-p^{1,2}\right)\right)^{2}+\mu\left|\varepsilon^{1,2}-p^{1,2}\right|^{2}+\frac{k_{\text {hard }}}{2}\left(\left|p^{1,2}\right|^{2}+\left(p_{11}+p_{22}\right)^{2}\right) .
\end{aligned}
$$

We define the limit energy $\mathcal{E}^{0}:[0, T] \times \mathcal{Q} \rightarrow \mathbb{R}_{\infty}$ by

$$
\mathcal{E}^{0}(t, q):= \begin{cases}\int_{\Omega} \overline{\mathbb{W}}\left(\varepsilon^{1,2}(u), p^{1,2}\right) \mathrm{d} x-\langle\ell(t), u\rangle, & \text { if } q \in \mathcal{Q}_{\mathrm{KL}} . \\ +\infty, & \text { else }\end{cases}
$$

For the limit dissipation functional the derivation is even simpler. We immediately see that $\mathcal{R}^{0}: \mathcal{P} \rightarrow \mathbb{R}_{\infty}$ defined by

$$
\mathcal{R}^{0}(\dot{p})= \begin{cases}\sigma_{\text {yield }} \int_{\Omega}\left|\dot{p}-\left(\dot{p}_{11}+\dot{p}_{22}\right) e_{3} \otimes e_{3}\right| \mathrm{d} x, & \text { if } \dot{p} \in \mathcal{P}_{\mathrm{KL}} \\ +\infty, & \text { else },\end{cases}
$$

is the $\Gamma$-limit, where $e_{3}=(0,0,1)^{\top}$.

The following convergence result, which is the central aim of this paper, shows that the solutions $q^{h}=\left(u^{h}, p^{h}\right)$ of the RIS $\left(\mathcal{Q}, \mathcal{E}^{h}, \mathcal{R}^{h}\right)$ converge, for $h \rightarrow 0$, to solutions $q=(u, p)$ of the limiting RIS $\left(\mathcal{Q}, \mathcal{E}^{0}, \mathcal{R}^{0}\right)$. The proof will be established in Sect. 3. We follow the ideas in [13] and adapt the results presented therein to our needs. The specific properties of the limit system as well as the connection with the model derived in [11] are discussed in Sect. 4.

Theorem 2.2. Assume that the $\operatorname{RIS}\left(\mathcal{Q}, \mathcal{E}^{h}, \mathcal{R}^{h}\right)$ are given as above for all $h \in[0,1]$. Consider a family of solutions $q^{h}:[0, T] \rightarrow \mathcal{Q}$, as defined in (2.3). Moreover assume that we have $\mathcal{E}^{h}\left(0, q_{0}^{h}\right) \rightarrow \mathcal{E}^{0}\left(0, q_{0}^{0}\right)$ and $q_{0}^{h} \rightarrow q_{0}^{0}$ in $\mathcal{Q}$. Then for all $t \in[0, T]$ we have the convergences 


$$
q^{h}(t) \rightarrow q(t), \quad \mathcal{E}^{h}\left(t, q^{h}(t)\right) \rightarrow \mathcal{E}^{0}(t, q(t)), \quad \int_{0}^{t} \mathcal{R}^{h}\left(\dot{q}^{h}\right) \mathrm{d} t \rightarrow \int_{0}^{t} \mathcal{R}^{0}(\dot{q}) \mathrm{d} t .
$$

Moreover, $q$ is an energetic solution of the $\operatorname{RIS}\left(\mathcal{Q}, \mathcal{E}^{0}, \mathcal{R}^{0}\right)$.

Remark 2.3. Note that the existence of initial data $q_{0}^{h}$ satisfying the assumptions in Theorem 2.2 is not trivial. We refer to [11] for a discussion of this question.

\section{Proof of Theorem 2.2}

In this section we will prove our main result which is stated in terms of $\Gamma$-convergence of the energy functionals $\mathcal{E}^{h}$ and the dissipation functionals $\mathcal{R}^{h}$. We will use the weak and the strong topologies in the underlying Hilbert space $\mathcal{Q}$. More specifically we use the notion of Mosco convergence (cf. [2,5]) denoted by $\mathcal{I}_{n} \stackrel{\mathrm{M}}{\longrightarrow} \mathcal{I}$. The definition is as follows

$$
\mathcal{I}_{n} \stackrel{\mathrm{M}}{\longrightarrow} \mathcal{I} \Longleftrightarrow\left\{\begin{aligned}
\text { (i) } & \text { Liminf estimate: } \\
& q_{n} \rightarrow q \Longrightarrow \\
\text { (ii) } & \text { Limsup estimate (existence of recovery sequences) } \\
& \forall \widehat{q} \in \mathcal{Q} \exists\left(\widehat{q}_{n}\right)_{n}: \widehat{q}_{n} \rightarrow \widehat{q} \text { and } \mathcal{I}(\widehat{q}) \geq \limsup _{n \rightarrow \infty} \mathcal{I}_{n}\left(\widehat{q}_{n}\right) .
\end{aligned}\right.
$$

Hence, Mosco convergence is nothing but $\Gamma$-convergence with respect to both the weak and strong topology.

In the following we will use the notation $[n]=\{1, \ldots, n\}, n \in \mathbb{N}$ in order to shorten notation.

\section{1. $\Gamma$-limit of the energy functional}

Proposition 3.1. Let $\mathcal{E}^{h}:[0, T] \times \mathcal{Q} \rightarrow \mathbb{R}_{\infty}$ and $\mathcal{E}^{0}:[0, T] \times \mathcal{Q} \rightarrow \mathbb{R}_{\infty}$ be defined as above. Then $\mathcal{E}^{h} \stackrel{\Gamma}{\rightarrow} \mathcal{E}^{0}$ as $h \rightarrow 0$ with respect to the weak topology on $\mathcal{Q}$. Indeed, $\mathcal{E}^{h}$ even converges to $\mathcal{E}^{0}$ in the sense of Mosco convergence.

The proof is similar to the classical one by Ciarlet [4, Sect. 1.4 and 1.11].

Proof. (i) We start by proving the liminf inequality. Let $\left(h_{n}\right)_{n \in \mathbb{N}}$ be a vanishing sequence. To simplify notation we will replace $\left(h_{n}\right)$ by $n$ whenever no confusion can arise. Assume that $q=(u, p) \in \mathcal{Q}$ and $q^{n}=\left(u^{n}, p^{n}\right) \in \mathcal{Q}$ are such that $q^{n} \rightarrow q$ in $\mathcal{Q}$. If $q \notin \mathcal{Q}_{\mathrm{KL}}$ then there exists an index $i \in[3]$ such that either $\varepsilon_{i 3}(u) \neq 0$ or $p_{i 3} \neq 0$. Notice that by (2.6) we have for any $C>0$ the estimate

$$
\begin{aligned}
\mathcal{E}^{n}\left(t, q^{n}\right) & \geq c\left(\left\|S_{n} \varepsilon\left(u^{n}\right) S_{n}\right\|_{\mathrm{L}^{2}}^{2}+\left\|S_{n} p^{n} S_{n}\right\|_{\mathrm{L}^{2}}^{2}\right)-\left\langle\ell\left(t^{n}\right), u^{n}\right\rangle \\
& \geq C\left(\left\|\varepsilon\left(u^{n}\right)_{i 3}\right\|_{\mathrm{L}^{2}}^{2}+\left\|p_{i 3}^{n}\right\|_{\mathrm{L}^{2}}^{2}\right)-\left\langle\ell(t), u^{n}\right\rangle
\end{aligned}
$$

for sufficiently large $n$. Therefore, we deduce that

$$
\liminf _{n \rightarrow \infty} \mathcal{E}^{n}\left(t, w^{n}\right) \geq C\left(\left\|\varepsilon_{i 3}(u)\right\|_{\mathrm{L}^{2}}^{2}+\left\|p_{i 3}\right\|_{\mathrm{L}^{2}}^{2}\right)-\|\ell\|_{\mathrm{L}^{\infty}\left(0, T ; H^{*}\right)}\|u\|_{\mathrm{H}^{1}},
$$


where $C$ can be chosen arbitrary large and obtain $\mathcal{E}^{n}\left(t, q^{n}\right) \rightarrow \infty$. For $q \in \mathcal{Q}_{\mathrm{KL}}$ we proceed as follows. First we compute

$$
\begin{aligned}
\mathcal{E}^{n}\left(t, q^{n}\right)= & \int_{\Omega} \overline{\mathbb{W}}\left(\varepsilon^{1,2}\left(u^{n}\right),\left(p^{n}\right)^{1,2}\right) \mathrm{d} x-\left\langle\ell(t), u^{n}\right\rangle \\
& +\frac{1}{h_{n}^{2}} \int_{\Omega} 2 \mu \sum_{i=1}^{2}\left(\varepsilon_{i 3}\left(u^{n}\right)-p_{i 3}^{n}\right)^{2}+k_{\text {hard }} \sum_{i=1}^{2}\left(p_{i 3}^{n}\right)^{2} \mathrm{~d} x \\
& +\int_{\Omega}(\lambda+2 \mu)\left(\frac{\lambda}{\lambda+2 \mu} \sum_{i=1}^{2}\left(\varepsilon_{i i}\left(u^{n}\right)-p_{i i}^{n}\right)+\frac{1}{h_{n}^{2}}\left(\varepsilon_{33}\left(u^{n}\right)-p_{33}^{n}\right)\right)^{2} \mathrm{~d} x
\end{aligned}
$$

Note, that we can always assume that $p_{33} / h_{n}^{2}+p_{11}+p_{22}=0$ since $\mathcal{E}^{n}\left(t^{n}, q^{n}\right)=$ $\infty$ otherwise. Hence, we especially have

$$
\sum_{i, j=1}^{2}\left(p_{i j}^{n}\right)^{2}+\left(p_{33}^{n} / h_{n}^{2}\right)^{2}=\sum_{i, j=1}^{2}\left(p_{i j}^{n}\right)^{2}+\left(p_{11}^{n}+p_{22}^{n}\right)^{2} .
$$

Using that the terms in the second and third line of (3.2) are positive we obtain

$$
\mathcal{E}^{n}\left(t, q^{n}\right) \geq \int_{\Omega} \overline{\mathbb{W}}\left(\varepsilon^{1,2}\left(u^{n}\right),\left(p^{n}\right)^{1,2}\right) \mathrm{d} x-\left\langle\ell(t), u^{n}\right\rangle .
$$

The right-hand side is weakly lower semi-continuous on $\mathcal{Q}$ and as $n \rightarrow \infty$ we obtain

$$
\liminf _{n \rightarrow \infty} \mathcal{E}^{n}\left(t, q^{n}\right) \geq \mathcal{E}^{0}(t, q)
$$

(ii) It remains to construct a recovery sequence for $q \in \mathcal{Q}_{\mathrm{KL}}$. For this we choose $q^{n}$ to be the unique solution of the elliptic problem

$$
\left\langle A^{n} q^{n}, \widetilde{q}\right\rangle=\left\langle\ell_{q}, \widetilde{q}\right\rangle:=\left\langle A^{0} q, \widetilde{q}\right\rangle \quad \forall \widetilde{q} \in \mathcal{Q}^{h_{n}},
$$

where $A^{n}: \mathcal{Q}^{h_{n}} \rightarrow \mathcal{Q}^{h_{n}}$ and $A^{0}: \mathcal{Q} \rightarrow \mathcal{Q}^{*}$ are the linear and self-adjoint operators associated with the quadratic energies $\mathcal{E}^{h}$ and $\mathcal{E}^{0}$, i.e.,

$$
A^{h}=\mathrm{D}_{q} \mathcal{E}^{h} \text { on } \mathcal{Q}^{h} \text { and } A^{0}=\mathrm{D}_{q} \mathcal{E}^{0} \text { on } \mathcal{Q}_{\mathrm{KL}} .
$$

Due to the Lax-Milgram theorem $q^{n}$ exists and is uniquely determined. We want to show that $q^{n}$ is a recovery sequence for $q$. To this end we use (2.6) and obtain that both $\boldsymbol{a}^{n}:=S_{n} \varepsilon\left(u^{n}\right) S_{n}$ and $\boldsymbol{b}^{n}:=S_{n} p^{n} S_{n}$ are uniformly bounded in $\mathrm{L}^{2}\left(\Omega ; \mathbb{R}_{\mathrm{sym}}^{3 \times 3}\right)$ and therefore also $u^{n}$ and $p^{n}$ in $\mathcal{U}$ and $\mathcal{P}$, respectively. Hence we can extract a (not relabelled) subsequence $q^{n}$ such that $q^{n} \rightarrow \widehat{q}$. Moreover, we know that $\widehat{q}=(\widehat{u}, \widehat{p}) \in \mathcal{Q}_{\mathrm{KL}}$. Choosing a further (also not relabelled) subsequence we have that $\boldsymbol{a}_{n} \rightarrow \boldsymbol{a}$ and $\boldsymbol{b}_{n} \rightarrow \boldsymbol{b}$ in $\mathrm{L}^{2}\left(\Omega ; \mathbb{R}_{\mathrm{sym}}^{3 \times 3}\right)$. It holds that $\boldsymbol{a}_{i j}=\varepsilon_{i j}(\widehat{u})$ and $\boldsymbol{b}_{i j}=\widehat{p}_{i j}$ for $i, j \in[2]$. We set $\boldsymbol{c}^{n}:=\boldsymbol{a}^{n}-\boldsymbol{b}^{n}$ such that $c^{n} \rightarrow c:=a-b$.

For an arbitrary $v \in \mathcal{U}$ we set $\widetilde{q}=(v, 0)$ in (3.3) and obtain

$$
\begin{aligned}
& \left\langle\ell_{q}, \widetilde{q}\right\rangle \\
& =\int_{\Omega} \lambda\left(\operatorname{tr} c^{n}\right)\left(\varepsilon_{11}(v)+\varepsilon_{22}(v)+\frac{1}{h_{n}^{2}} \varepsilon_{33}(v)\right)+2 \mu \boldsymbol{c}^{n}:\left(S_{n} \varepsilon(v) S_{n}\right) \mathrm{d} x
\end{aligned}
$$


We now choose $v$ such that $v_{1}=v_{2}=0$ and multiplicate with $h_{n}^{2}$. After passing to the limit we obtain for all $v_{3} \in \mathrm{H}_{\Gamma_{0}}^{1}(\Omega)$

$$
\int_{\Omega}\left(\lambda\left(\boldsymbol{c}_{11}+\boldsymbol{c}_{22}\right)+(\lambda+2 \mu) \boldsymbol{c}_{33}\right) \partial_{x_{3}} v_{3} \mathrm{~d} x=0 .
$$

Thus, we have the following identity (see [4, Proof of Theorem 1.4])

$$
c_{33}=-\frac{\lambda}{2 \mu+\lambda}\left(c_{11}+c_{22}\right)
$$

Analogously, by setting $v_{3}=0$ in (3.4) and using the symmetry of $\boldsymbol{c}^{n}$ we get

$$
4 \mu \int_{\Omega} \boldsymbol{c}_{13}^{n} \partial_{x_{3}} v_{1}+c_{23}^{n} \partial_{x_{3}} v_{2} \mathrm{~d} x=h_{n}\left\langle\ell_{q}, \widetilde{q}\right\rangle .
$$

After passing to the limit we deduce that $\boldsymbol{c}_{13}=\boldsymbol{c}_{23}=0$.

Since $q^{n} \in \mathcal{Q}^{h_{n}}$ implies $p_{33}^{n} / h_{n}^{2}+p_{11}^{n}+p_{22}^{n}=0$ we directly infer that $p_{33}^{n} / h_{n}^{2} \rightarrow-\widehat{p}_{11}-\widehat{p}_{22}=\boldsymbol{b}_{33}$. Therefore we deduce

$$
\boldsymbol{a}_{33}=-\widehat{p}_{11}-\widehat{p}_{22}-\frac{\lambda}{\lambda+2 \mu} \sum_{i=1}^{2}\left(\varepsilon_{i i}(\widehat{u})-\widehat{p}_{i i}\right) .
$$

In a next step we set $\widetilde{q}=(0, \widetilde{p})$ with $\widetilde{p} \in \mathcal{P}^{h_{n}}$ such that $\widetilde{p}_{i j}=0$ for $i, j \in[2]$. By plugging it into (3.3) we derive

$$
\int_{\Omega} 2 \mu\left(\boldsymbol{c}_{i 3}^{n} \widetilde{p}_{i 3}+\boldsymbol{c}_{3 i}^{n} \widetilde{p}_{3 i}\right)+2 k_{\mathrm{hard}}\left(\boldsymbol{b}_{i 3}^{n} \widetilde{p}_{i 3}+\boldsymbol{b}_{3 i}^{n} \widetilde{p}_{3 i}\right) \mathrm{d} x=0 .
$$

By passing to the limit and using that $\boldsymbol{c}_{3 i}=\boldsymbol{c}_{i 3}=0$ we obtain $\boldsymbol{b}_{3 i}=\boldsymbol{b}_{i 3}=0$ and thus also $\boldsymbol{a}_{3 i}=\boldsymbol{a}_{i 3}=0$ for $i \in[2]$. For an arbitrary $\widetilde{q}=(\widetilde{u}, \widetilde{p}) \in \mathcal{Q}_{\mathrm{KL}}$ we define the sequence $\widetilde{q}^{n}=\left(\widetilde{u}^{n}, \widetilde{p}^{n}\right) \in \mathcal{Q}^{h_{n}}$ by $\widetilde{u}^{n}=\widetilde{u}, \widetilde{p}_{i j}^{n}=\widetilde{p}_{i j}$ for $(i, j) \neq(3,3)$ and $\widetilde{p}_{33}^{n}=-h_{n}^{2}\left(\widetilde{p}_{11}+\widetilde{p}_{22}\right)$. Then $\widehat{q}^{n} \rightarrow \widehat{q}$ strongly in $\mathcal{Q}$ and

$$
0=\lim _{n \rightarrow \infty}\left\langle A^{n} q^{n}, \widetilde{q}^{n}\right\rangle-\left\langle\ell_{q}, \widetilde{q}^{n}\right\rangle=\left\langle A^{0} \widehat{q}, \widetilde{q}\right\rangle-\left\langle\ell_{q}, \widetilde{q}\right\rangle=0
$$

Therefore, it holds that $A^{0}(\widehat{q}-q)=0$ in $\mathcal{Q}_{\mathrm{KL}}^{*}$, which yields $\widehat{q}=q$. Hence, we have that $q^{n} \rightarrow q$ in $\mathcal{Q}$. It remains to show that the energies converge. To this end note that by means of $\boldsymbol{a}^{n}, \boldsymbol{a}$ and $\boldsymbol{b}^{n}, \boldsymbol{b}$ and the identity in (3.5) we can write

$$
\begin{aligned}
\left\langle A^{0} q, q\right\rangle & =\int_{\Omega} \mathcal{C}(\boldsymbol{a}-\boldsymbol{b}):(\boldsymbol{a}-\boldsymbol{b})+k_{\mathrm{hard}}|\boldsymbol{b}|^{2} \mathrm{~d} x \\
\left\langle A^{n} q^{n}, q^{n}\right\rangle & =\int_{\Omega} \mathcal{C}\left(\boldsymbol{a}^{n}-\boldsymbol{b}^{n}\right):\left(\boldsymbol{a}^{n}-\boldsymbol{b}^{n}\right)+k_{\mathrm{hard}}\left|\boldsymbol{b}^{n}\right|^{2} \mathrm{~d} x,
\end{aligned}
$$


where $\mathcal{C}$ is the elasticity tensor defined in Sect. 2.1. Hence, using the quadratic structure of the energy functionals we compute

$$
\begin{aligned}
& c\left(\left\|\boldsymbol{a}^{n}-\boldsymbol{a}\right\|_{\mathrm{L}^{2}}^{2}+\left\|\boldsymbol{b}^{n}-\boldsymbol{b}\right\|_{\mathrm{L}^{2}}^{2}\right) \\
& \leq \int_{\Omega} \mathcal{C}\left(\boldsymbol{c}^{n}-(\boldsymbol{a}-\boldsymbol{b})\right):\left(\boldsymbol{c}^{n}-(\boldsymbol{a}-\boldsymbol{b})\right)+k_{\mathrm{hard}}\left|\boldsymbol{b}^{n}-\boldsymbol{b}\right|^{2} \mathrm{~d} x \\
& =\left\langle A^{0} q, q\right\rangle-2 \int_{\Omega} \mathcal{C}(\boldsymbol{a}-\boldsymbol{b}): \boldsymbol{c}^{n}+k_{\mathrm{hard}} \boldsymbol{b}: \boldsymbol{b}^{n} \mathrm{~d} x+\left\langle A^{n} q^{n}, q^{n}\right\rangle \\
& =\left\langle A^{0} q, q\right\rangle-2 \int_{\Omega} \mathcal{C}(\boldsymbol{a}-\boldsymbol{b}): \boldsymbol{c}^{n}+k_{\mathrm{hard}} \boldsymbol{b}: \boldsymbol{b}^{n} \mathrm{~d} x+\left\langle\ell_{q}, q^{n}\right\rangle \\
& \quad \rightarrow\left\langle\ell_{q}, q\right\rangle-\left\langle A^{0} q, q\right\rangle=0 .
\end{aligned}
$$

Therefore, we obatin the strong convergences $\boldsymbol{a}^{n} \rightarrow \boldsymbol{a}$ and $\boldsymbol{b}^{n} \rightarrow \boldsymbol{b}$ in $\mathrm{L}^{2}\left(\Omega ; \mathbb{R}_{\mathrm{sym}}^{3 \times 3}\right)$ and consequently $q^{n} \rightarrow q$ strongly in $\mathcal{Q}$. To finish the proof note that

$$
\mathcal{E}^{n}\left(t, q^{n}\right)=\frac{1}{2}\left\langle A^{n} q^{n}, q^{n}\right\rangle-\left\langle\ell(t), u^{n}\right\rangle \longrightarrow \frac{1}{2}\left\langle A^{0} q, q\right\rangle-\langle\ell(t), u\rangle=\mathcal{E}^{0}(t, q)
$$

where we used the strong convergence of $\boldsymbol{a}^{n}$ and $\boldsymbol{b}^{n}$ and (3.6).

\section{2. $\Gamma$-limit of the dissipation functional}

Proposition 3.2. Let $\mathcal{R}^{h}$ and $\mathcal{R}^{0}$ be defined as above. Then $\mathcal{R}^{h} \stackrel{\Gamma}{\rightarrow} \mathcal{R}^{0}$ with respect to the weak topology on $\mathcal{P}$. What is more, $\mathcal{R}^{h}$ converges to $\mathcal{R}^{0}$ in the sense of Mosco convergence, too.

Proof. (i) Let $\left(h^{n}\right)_{n \in \mathbb{N}}$ be a vanishing sequence, choose $\dot{p}^{n}$ such that $\dot{p}^{n} \rightarrow \dot{p}$ Assume that $\dot{p} \notin \mathcal{P}_{\mathrm{KL}}$. Then there exists some $i \in[3]$ such that $\dot{p}_{i 3} \neq 0$. For sufficiently large $n$ we have

$$
\mathcal{R}^{n}\left(\dot{p}^{n}\right)=\sigma_{\text {yield }} \int_{\Omega}\left|S_{n} \dot{p}^{n} S_{n}\right| \mathrm{d} x \geq \frac{\sigma_{\text {yield }}}{h_{n}} \int_{\Omega}\left|\dot{p}_{i 3}^{n}\right| \mathrm{d} x \geq C \sigma_{\text {yield }} \int_{\Omega}\left|\dot{p}_{i 3}^{n}\right| \mathrm{d} x,
$$

where $C$ can be chosen arbitrarily large. Due to the lower semicontinuity of the norm we obtain $\mathcal{R}^{n}\left(\dot{p}^{n}\right) \rightarrow+\infty$.

Now let $\dot{p} \in \mathcal{P}_{\mathrm{KL}}$. We can safely assume that $\operatorname{tr}\left(S_{n} \dot{p}^{n} S_{n}\right)=0$ for all $n$. We have that

$$
\mathcal{R}^{n}\left(\dot{p}^{n}\right)=\sigma_{\text {yield }} \int_{\Omega}\left|S_{n} \dot{p}^{n} S_{n}\right| \mathrm{d} x \geq \sigma_{\text {yield }} \int_{\Omega} \sqrt{\sum_{i, j=1}^{2}\left(\dot{p}_{i j}^{n}\right)^{2}+\left(\dot{p}_{11}^{n}+\dot{p}_{22}^{n}\right)^{2}} \mathrm{~d} x .
$$

The weak lower semicontinuity of the right-hand side grants the liminfinequality.

(ii) To construct a recovery sequence for a given $\dot{p} \in \mathcal{P}_{\mathrm{KL}}$ set $\dot{p}_{i j}^{n}=\dot{p}_{i j}$ for $(i, j) \neq(3,3)$ and $\dot{p}_{33}^{n}=-h_{n}^{2}\left(\dot{p}_{11}+\dot{p}_{22}\right)$. Note that $\operatorname{tr}\left(S_{n} p^{n} S_{n}\right)=0$ and therefore

$$
\mathcal{R}^{n}\left(\dot{p}^{n}\right)=\sigma_{\text {yield }} \int_{\Omega}\left|\dot{p}-\left(\dot{p}_{11}+\dot{p}_{22}\right) e_{3} \otimes e_{3}\right| \mathrm{d} x=\mathcal{R}^{0}(\dot{p}) .
$$

The last part of the assertion follows from the strong convergence $\dot{p}^{n} \rightarrow \dot{p}$ in $\mathcal{P}$. 
Note that for absolutely continuous functions $q:[0, T] \rightarrow \mathcal{Q}$ and $0 \leq r<$ $s \leq T$ we have

$$
\operatorname{Diss}_{h}(q ;[r, s])=\int_{r}^{s} \mathcal{R}^{h}(\dot{q}(t)) \mathrm{d} t,
$$

where we used the notation

$\operatorname{Diss}^{h}(q ;[r, s]):=\sup \left\{\sum_{i=1}^{n} \mathcal{R}^{h}\left(q\left(t_{i}\right)-q\left(t_{i-1}\right)\right): n \in \mathbb{N}, r \leq t_{0}<\cdots<t_{n} \leq s\right\}$.

which is defined for all pointwise defined functions. Using the lim inf estimate from $\mathcal{R}^{h} \stackrel{\mathrm{M}}{\longrightarrow} \mathcal{R}^{0}$ it is standard to show that $\operatorname{Diss}^{h}$ is lower semicontinuous in the sense that

$$
\begin{aligned}
& \left(\forall t \in[0, T]: q^{h}(t) \rightarrow q(t)\right) \\
& \quad \Rightarrow \operatorname{Diss}^{0}\left(q_{0} ;[0, T]\right) \leq \liminf _{h \rightarrow 0} \operatorname{Diss}^{h}\left(q^{h} ;[0, T]\right) .
\end{aligned}
$$

\subsection{Convergence of the solutions}

The main challenge is to establish the upper semicontinuity of the stable sets, i.e., limits of stable sequences remain stable with respect to the limit energy and dissipation functional. We have the following crucial result:

Proposition 3.3. Let $q^{n}$ be such that the stability condition w.r.t. $\left(\mathcal{E}^{n}, \mathcal{R}^{n}\right)$ is satisfied for $t \in[0, T]$. If $q^{n} \rightarrow q$ in $\mathcal{Q}$, then we have that $q$ satisfies the stability condition w.r.t. $\left(\mathcal{E}^{0}, \mathcal{R}^{0}\right)$.

Proof. In the following the time $t$ is fixed. We make use of the quadratic form of the energy functionals. In this case the global stability condition is equivalent to local stability condition $\ell(t)-A^{n} q^{n} \in \partial \mathcal{R}^{n}(0)$, where $A^{n}$ is the operator associated with $\mathcal{E}^{n}$ defined in the proof of Proposition 3.1. Hence, we want to show that

$$
\left\langle A^{0} q-\ell(t), \widehat{q}\right\rangle+\mathcal{R}^{0}(\widehat{q}) \geq 0 \quad \forall \widehat{q} \in \mathcal{Q}_{\mathrm{KL}},
$$

where $A^{0}$ is the operator associated with the limit energy. To this end we are going to show that for all $\widehat{q} \in \mathcal{Q}_{\mathrm{KL}}$ and for all stable sequences $q^{n}$ such that $q^{n} \rightarrow q$ we can construct a sequence $\widehat{q}^{n}$ such that $\mathcal{R}^{n}\left(\widehat{q}^{n}\right) \rightarrow \mathcal{R}^{0}(\widehat{q})$ and

$$
\left\langle A^{n} q^{n}, \widehat{q}^{n}\right\rangle \rightarrow\left\langle A^{0} q, \widehat{q}\right\rangle .
$$

Step 1. Let $q^{n}=\left(u^{n}, p^{n}\right)$ be such a weakly converging stable sequence. In particular, there exists a constant $C \geq 0$ such that $\mathcal{E}^{n}\left(t, q^{n}\right) \leq C$. As in the proof of Proposition 3.1 we obtain that

$$
\left\|S_{n} \varepsilon\left(u^{n}\right) S_{n}\right\|_{2}^{2}+\left\|S_{n} p^{n} S_{n}\right\|_{2}^{2} \leq C
$$

which directly implies $\varepsilon_{i 3}(u)=\varepsilon_{3 i}(u)=p_{i 3}=p_{3 i}=0$ for $i \in[3]$; hence $q \in \mathcal{Q}_{\mathrm{KL}}$. Moreover, we have that $p_{11}^{n}+p_{22}^{n}+p_{33}^{n} / h_{n}^{2}=0$ for all $n \in \mathbb{N}$ and therefore $p_{33}^{n} / h_{n}^{2} \rightarrow-p_{11}-p_{22}$. Let $\boldsymbol{a}^{n}:=S_{n} \varepsilon\left(u^{n}\right) S_{n}$ and $\boldsymbol{b}^{n}:=S_{n} p^{n} S_{n}$ as before. The estimate above allows us to extract weakly converging subsequences such that $\boldsymbol{a}^{n} \rightarrow \boldsymbol{a}$ and $\boldsymbol{b}^{n} \rightarrow \boldsymbol{b}$ in $\mathrm{L}^{2}\left(\Omega ; \mathbb{R}_{\mathrm{sym}}^{3 \times 3}\right)$. 
Step 2. If we test the global stability condition with $\left(u^{n} \pm \alpha v, p^{n}\right)$ for an arbitrary $v \in \mathcal{U}$ and let $\alpha \rightarrow 0$ we obtain

$$
\int_{\Omega} \lambda \operatorname{tr}\left(\boldsymbol{a}^{n}-\boldsymbol{b}^{n}\right) \cdot \operatorname{tr}\left(S_{n} \varepsilon(v) S_{n}\right)+2 \mu\left(\boldsymbol{a}^{n}-\boldsymbol{b}^{n}\right):\left(S_{n} \varepsilon(v) S_{n}\right) \mathrm{d} x=\left\langle\ell\left(t^{n}\right), v\right\rangle .
$$

Choosing $v=\left(0,0, v_{3}\right)^{\top}$ yields

$$
\boldsymbol{a}_{33}-\boldsymbol{b}_{33}=-\frac{\lambda}{2 \mu+\lambda}\left(\boldsymbol{a}_{11}-\boldsymbol{b}_{11}+\boldsymbol{b}_{11}-\boldsymbol{b}_{22}\right) \text {. }
$$

Since we know that $\boldsymbol{b}_{33}^{n}=p_{33}^{n} / h_{n}^{2} \rightarrow-p_{11}-p_{22}$ and $\boldsymbol{a}_{i j}^{n}=\varepsilon_{i j}\left(u^{n}\right) \rightarrow$ $\varepsilon_{i j}(u), i, j=1,2$, we obtain

$$
\boldsymbol{a}_{33}^{n}=\varepsilon\left(u^{n}\right)_{33} / h_{n}^{2} \rightarrow-p_{11}-p_{22}-\frac{\lambda}{2 \mu+\lambda}\left(\varepsilon_{11}(u)-p_{11}+\varepsilon_{22}(u)-p_{22}\right) \text {. }
$$

Step 3. Now, we take $\widehat{q}^{n}=\left(\widehat{u}^{n}, \widehat{p}^{n}\right)$ as the recovery sequence for $\widehat{q}$ as constructed in the proof of Proposition 3.1, i.e.,

$$
\widehat{q}^{n} \longrightarrow \widehat{q} \text { and } \mathcal{E}^{n}\left(t, \widehat{q}^{n}\right) \longrightarrow \mathcal{E}^{0}(t, \widehat{q})
$$

Note, that we have the strong convergence $\widehat{q}^{n} \rightarrow \widehat{q}$ in $\mathcal{Q}$. Moreover, we know that $\widehat{\boldsymbol{a}}^{n}:=S_{n} \varepsilon\left(\widehat{u}^{n}\right) S_{n}$ and $\widehat{\boldsymbol{b}}^{n}:=S_{n} \widehat{p}^{n} S_{n}$ converge strongly to $\widehat{\boldsymbol{a}}$ and $\widehat{\boldsymbol{b}}$ in $\mathrm{L}^{2}\left(\Omega ; \mathbb{R}_{\text {sym }}^{3 \times 3}\right)$, respectively. Here, we have that $\widehat{\boldsymbol{a}}_{i j}=\varepsilon_{i j}(\widehat{u})$ and $\widehat{\boldsymbol{b}}_{i j}=\widehat{p}_{i j}$ for $i, j=1,2$ and

$$
\begin{gathered}
\widehat{\boldsymbol{a}}_{13}=\widehat{\boldsymbol{a}}_{23}=\widehat{\boldsymbol{b}}_{13}=\widehat{\boldsymbol{b}}_{23}=0 \\
\widehat{\boldsymbol{b}}_{33}=-\widehat{p}_{11}-\widehat{p}_{22}, \quad \widehat{\boldsymbol{a}}_{33}=\widehat{\boldsymbol{b}}_{33}-\frac{\lambda}{2 \mu+\lambda}\left(\widehat{\boldsymbol{a}}_{11}-\widehat{\boldsymbol{b}}_{11}+\widehat{\boldsymbol{a}}_{22}-\widehat{\boldsymbol{b}}_{22}\right) .
\end{gathered}
$$

Step 4. In order to prove (3.11) we write the product in the following way

$$
\begin{aligned}
& \left\langle A^{n} q^{n}, \widehat{q}\right\rangle \\
& \quad=\int_{\Omega} \lambda \operatorname{tr}\left(\boldsymbol{a}^{n}-\boldsymbol{b}^{n}\right) \cdot \operatorname{tr}\left(\widehat{\boldsymbol{a}}^{n}-\widehat{\boldsymbol{b}}^{n}\right)+2 \mu\left(\boldsymbol{a}^{n}-\boldsymbol{b}^{n}\right):\left(\widehat{\boldsymbol{a}}^{n}-\widehat{\boldsymbol{b}}^{n}\right)+k_{\mathrm{hard}} \boldsymbol{b}^{n}: \widehat{\boldsymbol{b}}^{n} \mathrm{~d} x
\end{aligned}
$$

Using the weak and the strong convergence of $\boldsymbol{a}^{n}, \boldsymbol{b}^{n}$ and $\widehat{\boldsymbol{a}}^{n}, \widehat{\boldsymbol{b}}^{n}$, respectively, yields

$$
\begin{aligned}
\left\langle A^{n} q^{n}, \widehat{q}^{n}\right\rangle & \rightarrow \int_{\Omega} \lambda \operatorname{tr}(\boldsymbol{a}-\boldsymbol{b}) \cdot \operatorname{tr}(\widehat{\boldsymbol{a}}-\widehat{\boldsymbol{b}})+2 \mu(\boldsymbol{a}-\boldsymbol{b}):(\widehat{\boldsymbol{a}}-\widehat{\boldsymbol{b}})+k_{\mathrm{hard}} \boldsymbol{b}: \widehat{\boldsymbol{b}} \mathrm{d} x \\
& =\left\langle A^{0} q, \widehat{q}\right\rangle,
\end{aligned}
$$

where we have used the relations derived in Step 2. Thus, we have shown (3.11).

Step 5. It remains to show that $\mathcal{R}^{n}\left(\widehat{p}^{n}\right) \rightarrow \mathcal{R}^{0}(\widehat{p})$, i.e., $\widehat{p}^{n}$ is also a recovery sequence w.r.t. $\mathcal{R}^{h}$. For this, note that we have

$$
\mathcal{R}^{n}\left(\widehat{p}^{n}\right)=\sigma_{\text {yield }} \int_{\Omega}\left|\widehat{\boldsymbol{b}}^{n}\right| \mathrm{d} x .
$$


Hence, the convergence of $\mathcal{R}^{n}\left(\widehat{p}^{n}\right)$ follows directly from the strong convergence of $\widehat{\boldsymbol{b}}^{n}$.

We are now in position to provide the full proof of the $\Gamma$-convergence result for the quadratic rate-independent systems $\left(\mathcal{Q}, \mathcal{E}^{h}, \mathcal{R}^{h}\right)$.

Proof of Theorem 2.2. We will mainly follow the same six steps of the argument as in [13].

Step 1: A priori estimates. Using Theorem 2.1 we obtain the uniform bounds

$$
\left\|q^{h}\right\|_{\mathrm{C}^{0}([0, T] ; \mathcal{Q})}+\left\|\dot{q}^{h}\right\|_{\mathrm{L}^{1}(0, T ; \mathcal{Q})} \leq C
$$

for all $h \in(0,1]$.

Step 2: Selection of subsequences. Via the selection principle of ArzelaAscoli we find a $q^{*} \in \mathrm{C}^{0}(0, T ; \mathcal{Q})$ and a suitable subsequence $\left(h_{n}\right)_{n \in \mathbb{N}}$ such that $q^{h_{n}}(t) \rightarrow q_{*}(t)$ in $\mathcal{Q}$ for all $t \in[0, T]$. We can estimate

$$
\sum_{k=1}^{n}\left|q^{h}\left(t_{k}\right)-q^{h}\left(s_{k}\right)\right| \leq \sum_{k=1}^{n} \int_{s_{k}}^{t_{k}}\left|\dot{q}^{h}(t)\right|_{\mathcal{Q}} \mathrm{d} t \leq C \theta
$$

and using the weak lower semicontinuity of the norm we have that the limit $q_{*}$ is absolutely continuous as well.

Step 3: Stability of the limit. Since $q^{h_{n}}(t)$ is stable w.r.t. $\left(\mathcal{E}^{n}, \mathcal{R}^{n}\right)$ and $q^{h_{n}}(t) \rightarrow$ $q_{*}(t)$ we infer from Proposition 3.3 that $q_{*}(t)$ is also stable.

Step 4: Upper energy estimate. The energy balance for $q^{h}$ reads

$$
\mathcal{E}^{h}\left(t, q^{h}(t)\right)+\operatorname{Diss}^{h}\left(q^{h} ;[0, t]\right)=\mathcal{E}^{h}\left(0, q^{h}(0)\right)-\int_{0}^{t}\left\langle\dot{\ell}(s), q^{h}(s)\right\rangle \mathrm{d} s .
$$

Using the weak convergence of the solutions we can pass to the limit $h_{n} \rightarrow 0$ by employing the Mosco convergence of the energy functionals and (3.9). Moreover, using the dominated convergence theorem and the uniform boundedness of $u^{h}$ we obtain

$$
\int_{0}^{t}\left\langle\dot{\ell}(s), u^{h}(s)\right\rangle \mathrm{d} s \rightarrow \int_{0}^{t}\left\langle\dot{\ell}(s), u_{*}(s)\right\rangle \mathrm{d} s
$$

for all $t \in[0, T]$. This leads to the estimate

$$
\mathcal{E}^{0}\left(t, q_{*}(t)\right)+\operatorname{Diss}^{0}\left(q_{*},[0, t]\right) \leq \mathcal{E}^{0}\left(0, q_{*}(0)\right)-\int_{0}^{t}\left\langle\dot{\ell}(s), q_{*}(s)\right\rangle \mathrm{d} s .
$$

Here we use the liminf-estimates on the left-hand side, while convergences hold on the right-hand side.

Step 5: Lower energy estimate. It remains to show the opposite inequality. For this, note that $q_{*}$ is absolutely continuous and hence for any $\rho>0$ there exists some $\delta>0$ such that

$$
\forall t, s \in[0, T],|t-s|<\delta:\left|q_{*}(t)-q_{*}(s)\right| \leq \frac{\rho}{\|\dot{\ell}(t)\|_{L^{1}\left(0, T ; \mathcal{U}^{*}\right)}} .
$$


Choose $\left(r_{i}\right)_{i=0}^{N} \subset[0, t]$ with $r_{0}=0, r_{N}=t, r_{i}>r_{i-1}$ and $\left|r_{i}-r_{i-1}\right| \leq \delta$ for all $i \in[N]$. Then, by using the stability of the limit process $w$, we have that

$$
\begin{aligned}
\mathcal{E}( & \left.t, q_{*}(t)\right)+\operatorname{Diss}^{0}\left(q_{*} ;[0, t]\right) \\
\geq & \mathcal{E}^{0}\left(0, q_{*}(0)\right) \\
& +\sum_{i=1}^{N} \mathcal{E}^{0}\left(r_{i}, q_{*}\left(r_{i}\right)\right)+\mathcal{R}^{0}\left(q_{*}\left(r_{i}\right)-q_{*}\left(r_{i-1}\right)\right)-\mathcal{E}^{0}\left(r_{i-1}, q_{*}\left(r_{i-1}\right)\right) \\
\geq & \mathcal{E}^{0}\left(0, q_{*}(0)\right)+\sum_{i=1}^{N} \mathcal{E}^{0}\left(r_{i}, q_{*}\left(r_{i}\right)\right)-\mathcal{E}^{0}\left(r_{i-1}, q_{*}\left(r_{i}\right)\right) \\
= & \mathcal{E}^{0}\left(0, q_{*}(0)\right)+\sum_{i=1}^{N} \int_{r_{i-1}}^{r_{i}}\left\langle\dot{\ell}(t), q_{*}(t)\right\rangle \mathrm{d} t+\int_{r_{i-1}}^{r_{i}}\left\langle\dot{\ell}(t), q_{*}\left(r_{i}\right)-q_{*}(t)\right\rangle \mathrm{d} t \\
\geq & \int_{0}^{t} \partial_{t} \mathcal{E}^{0}\left(s, q_{*}(s)\right) \mathrm{d} s+\mathcal{E}^{0}\left(0, q_{*}(0)\right)-\rho .
\end{aligned}
$$

Since $\rho>0$ was arbitrary we have shown the desired lower energy estimate.

Together with the Steps 3 and 4 we conclude that $q_{*}$ is equal to the unique energetic solution $q$. Therefore, the whole sequence $q^{h}(t)$ converges weakly to $q(t)$ for all $t \in[0, T]$.

Step 6: Improved convergence. Since the energy equality holds we know that for all $t \in[0, T]$ we have that

$$
\begin{aligned}
& \limsup _{h \rightarrow 0} \operatorname{Diss}^{h}\left(q^{h} ;[0, t]\right) \\
& =\limsup _{h \rightarrow 0}\left[\mathcal{E}^{h}\left(0, q^{h}(0)\right)+\int_{0}^{t} \partial_{s} \mathcal{E}^{h}\left(s, q^{h}(s)\right) \mathrm{d} s-\mathcal{E}^{h}\left(t, q^{h}(t)\right)\right] \\
& \geq \mathcal{E}^{0}(0, q(0))+\int_{0}^{t} \partial_{s} \mathcal{E}^{0}(s, q(s)) \mathrm{d} s-\mathcal{E}^{0}(t, q(t)) \\
& =\operatorname{Diss}^{0}(q ;[0, t]) .
\end{aligned}
$$

Combining this estimate with (3.9) we obtain that for all $t \in[0, T]$

$$
\operatorname{Diss}^{h}\left(q^{h} ;[0, t]\right) \rightarrow \operatorname{Diss}^{0}(q ;[0, t]) \quad \text { and } \quad \mathcal{E}^{h}\left(t, q^{h}(t)\right) \rightarrow \mathcal{E}^{0}(t, q(t)) .
$$

Finally, since the energy functionals are equi-coercive on $\mathcal{Q}$ we get the pointwise strong convergence of $q^{h}$ as a consequence of the convergence of the energies by using a similar argument as in the proof of Proposition 3.1.

\section{Discussion of the elastoplastic plate model}

In this section we want to discuss how the limit model obtained in Sect. 2.3 can be reduced to a two-dimensional problem coupled to plastic effects that can either be described by a three-dimensional model with internal variables or by a vector-valued Prandtl-Ishlinskii operator associated to each point $x \in \omega$. 
The key point is that the Kirchhoff-Love displacements $u \in \mathcal{U}_{\mathrm{KL}}$ can be characterized by functions defined only on the midplane $\omega$ [see (2.8)]. Therefore, the limit energy $\mathcal{E}^{0}$ can be reduced by integrating over the variable $x_{3}$. In the following we will use the letter $y$ to indicate points in $\omega$. Furthermore, $\nabla$ and $\Delta$ denote the two-dimensional operators acting only on $y \in \omega$, i.e. $\nabla=\left(\partial_{y_{1}}, \partial_{y_{2}}\right)^{\top}, \Delta=\partial_{y_{1}}^{2}+\partial_{y_{2}}^{2}$, and $\mathrm{D}^{2} v_{3} \in \mathbb{R}_{\mathrm{sym}}^{2 \times 2}$ denotes the Hessian of $v_{3}: \omega \rightarrow \mathbb{R}$. Moreover we will use the two-dimensional in-plane strain tensor

$$
\varepsilon^{1,2}(v)=\frac{1}{2}\left(\nabla v^{1,2}+\left(\nabla v^{1,2}\right)^{\top}\right), \quad \text { where } \quad v^{1,2}=\left(v_{1}, v_{2}\right)^{\top},
$$

which does not depend on $v_{3}$.

Concerning the plastic strain variable $p \in \mathrm{L}^{2}\left(\Omega ; \mathbb{R}_{\mathrm{sym}}^{3 \times 3}\right)$ we will use the decomposition $\Omega=\omega \times(-1 / 2,1 / 2)$ and the identification

$$
\mathrm{L}^{2}\left(\omega \times(-1 / 2,1 / 2) ; \mathbb{R}_{\mathrm{sym}}^{3 \times 3}\right) \cong \mathrm{L}^{2}(\omega ; \mathbf{B}) \quad \text { with } \quad \mathbf{B}=\mathrm{L}^{2}\left((-1 / 2,1 / 2) ; \mathbb{R}_{\mathrm{sym}}^{3 \times 3}\right) .
$$

Thus, we associate with each point $y \in \omega$ an internal variable $p(y, \cdot) \in \mathbf{B}$. Using the isomorphism $\mathcal{K}$ between the space $\mathcal{U}_{\mathrm{KL}}$ and $V$ introduced in (2.8) we see that the rate-independent system $\left(\mathcal{Q}_{\mathrm{KL}}, \mathcal{E}^{0}, \mathcal{R}^{0}\right)$ is equivalent to the system $\left(\mathcal{Q}_{0}, \mathcal{E}_{0}, \mathcal{R}_{0}\right)$ with

$$
\begin{aligned}
& \mathcal{Q}_{0}:=V \times \mathrm{L}^{2}(\omega ; \mathbf{B}), \quad \mathcal{R}_{0}(\dot{p})=\int_{\omega} \int_{-1 / 2}^{1 / 2} R^{0}(\dot{p}) \mathrm{d} x_{3} \mathrm{~d} y \\
& \mathcal{E}_{0}(t, v, p)=\int_{\omega} W_{0}\left(\varepsilon^{1,2}(v), \mathrm{D}^{2} v_{3}, p\right) \mathrm{d} y-\left\langle\ell_{0}(t), v\right\rangle .
\end{aligned}
$$

The reduced energy-density $W_{0}$ can be decomposed into membrane, bending and plastic energy-densities, i.e.

$$
W_{0}(\varepsilon, D, p)=W_{\operatorname{memb}}\left(\varepsilon,\left[p^{1,2}\right]_{0}\right)+W_{\text {bend }}\left(D,\left[p^{1,2}\right]_{1}\right)+W_{\text {plast }}(p),
$$

where

$$
\begin{aligned}
W_{\text {memb }}(\varepsilon, \Pi) & =\frac{2 \lambda \mu}{\lambda+2 \mu}\left(\frac{1}{2}(\operatorname{tr} \varepsilon)^{2}-\operatorname{tr} \varepsilon \operatorname{tr} \Pi\right)+2 \mu\left(\frac{1}{2}|\varepsilon|^{2}-\varepsilon: \Pi\right), \\
W_{\text {bend }}(D, \Pi) & =\frac{2 \lambda \mu}{\lambda+2 \mu}\left(\frac{1}{48}(\operatorname{tr} D)^{2}+\operatorname{tr} D \operatorname{tr} \Pi\right)+2 \mu\left(\frac{1}{48}|D|^{2}+D: \Pi\right) \\
W_{\text {plast }}(p) & =\frac{\lambda \mu}{\lambda+2 \mu}\left\|\operatorname{tr} p^{1,2}\right\|_{2}^{2}+\mu\left\|p^{1,2}\right\|_{2}^{2}+\frac{k_{\text {hard }}}{2}\left(|p|^{2}+(\operatorname{tr} p)^{2}\right) .
\end{aligned}
$$

Here we used $\int_{-1 / 2}^{1 / 2} \mathrm{~d} x_{3}=1, \int_{-1 / 2}^{1 / 2} x_{3} \mathrm{~d} x_{3}=0$ and $\int_{-1 / 2}^{1 / 2} x_{3}^{2} \mathrm{~d} x_{3}=1 / 24$ and the short-hand notations

$$
[g]_{0}=\int_{-1 / 2}^{1 / 2} g\left(x_{3}\right) \mathrm{d} x_{3}, \quad[g]_{1}=\int_{-1 / 2}^{1 / 2} x_{3} g\left(x_{3}\right) \mathrm{d} x_{3}, \quad\|g\|_{2}^{2}=\int_{-1 / 2}^{1 / 2}\left|g\left(x_{3}\right)\right|^{2} \mathrm{~d} x_{3} .
$$


The reduced loading $\ell_{0}(t) \in V^{*}$ is given by

$$
\begin{aligned}
\left\langle\ell_{0}(t), v\right\rangle & =\int_{\omega} G_{\text {memb }}(t) \cdot v^{1,2}+g_{\text {bend }}(t) v_{3}+G_{\text {bend }}(t) \cdot \nabla v_{3} \mathrm{~d} y, \\
\text { where } & \\
G_{\text {memb }}(t, y) & =\left[F_{\text {vol }}^{1,2}(t, y, \cdot)\right]_{0}+F_{\text {surf }}^{1,2}(t, y, 1)+F_{\text {surf }}^{1,2}(t, y,-1), \\
g_{\text {bend }}(t, y) & =\left[F_{\text {vol } 3}(t, y, \cdot)\right]_{0}+F_{\text {surf } 3}(t, y, 1)+F_{\text {surf } 3}(t, y,-1), \\
G_{\text {bend }}(t, y) & =F_{\text {surf }}^{1,2}(t, y,-1)-F_{\text {surf }}^{1,2}(t, y, 1) .
\end{aligned}
$$

As in [11] the important structure in the form of the reduced energy-density $W_{0}$ is that the membrane strains $\varepsilon^{1,2}(v)$ only couple to the (even) averages $\left[p^{1,2}\right]_{0}$, while the bending strains $\mathrm{D}^{2} v_{3}$ only couple to the (odd) averages $\left[p^{1,2}\right]_{1}$. The energetic formulation of the derived evolutionary system $\left(\mathcal{Q}_{0}, \mathcal{E}_{0}, \mathcal{R}_{0}\right)$ is equivalent to the subdifferential formulation. It consists of two elliptic equations, one for the membrane part and one for the bending part, and the plastic flow rule. Both elliptic equations are nontrivially coupled to the plastic part (see $[10,11]$ for simple examples).

The strain tensors take the form

$$
\begin{aligned}
& \partial_{A} W_{\text {memb }}(A, D)=\Sigma_{0}(A-D) \in \mathbb{R}_{\mathrm{sym}}^{2 \times 2}, \\
& \partial_{B} W_{\text {bend }}(B, D)=\Sigma_{0}\left(\frac{1}{24} B-D\right) \in \mathbb{R}_{\text {sym }}^{2 \times 2},
\end{aligned}
$$

where $\Sigma_{0}(E):=\frac{2 \lambda \mu}{\lambda+2 \mu} \operatorname{tr} E I_{2}+2 \mu E$ is the reduced elasticity tensor.

In order to compute the subdifferential of $R^{0}$ we note that it can be written in the form $R^{0}(\dot{p})=\widetilde{R}^{0}(\mathbb{D} \dot{p})$, where

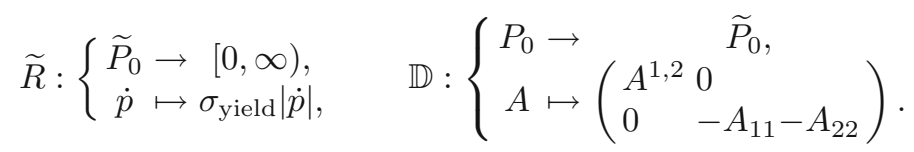

and

$$
\begin{aligned}
& P_{0}=\left\{A \in \mathbb{R}_{\mathrm{sym}}^{2 \times 2}: A_{13}=A_{23}=A_{33}=0\right\}, \\
& P_{0}=\left\{A \in \mathbb{R}_{\mathrm{dev}}^{3 \times 3}: A_{13}=A 23=0\right\} .
\end{aligned}
$$

Now, the subdifferential of $R^{0}$ can be calculated as follows by using the chain rule for subdifferentials (see [19, Theorem 23.9])

$$
\partial R^{0}(\dot{p})=\mathbb{D}^{*} \partial \widetilde{R}(\mathbb{D} \dot{p})=\mathbb{D}^{*} \begin{cases}\frac{\mathbb{D} \dot{p}}{|\mathbb{D} \dot{p}|} & \mathbb{D} \dot{p} \neq 0 \\ \sigma_{\text {yield }} B_{1}(0) & \text { otherwise }\end{cases}
$$

where $\mathbb{D}^{*}$ is the adjoint operator of $\mathbb{D}$ and $B_{1}(0)$ is the unit ball in $\widetilde{P}_{0}$ centered at 0 . A simple computation shows that for $\sigma \in P_{0}^{*}$ we have $\left(\mathbb{D}^{*}\right)^{-1} \sigma=\operatorname{dev} \sigma \in \widetilde{P}_{0}^{*}$. For $A \in \mathbb{R}_{\mathrm{sym}}^{2 \times 2}$ let $\llbracket A \| 0 \rrbracket \in \mathbb{R}_{\mathrm{sym}}^{3 \times 3}$ denote the enlargement of $A$ by 0 . We compute that the plastic stress $\sigma_{\mathrm{pl}}$ is given by

$$
\begin{aligned}
& \sigma_{\mathrm{pl}}=\mathrm{D}_{p} \mathcal{E}^{0}(t, v, p) \\
& =\llbracket \Sigma_{0}\left(p^{1,2}-\varepsilon^{1,2}(v)+x_{3} \mathrm{D}^{2} v_{3}\right)\left\|0 \rrbracket+k_{\text {hard }} \llbracket\left(\begin{array}{ll}
2 p_{11}+p_{22} & p_{12} \\
p_{23} & p_{11}+2 p_{22}
\end{array}\right)\right\| 0 \rrbracket .
\end{aligned}
$$


Hence, the subdifferential formulation for $\mathcal{E}^{0}$ and $\mathcal{R}^{0}$ is formally equivalent to

$$
\begin{aligned}
& 0=-\operatorname{div}\left(\Sigma_{0}\left(\varepsilon^{1,2}(v)-\left[p^{1,2}\right]_{0}\right)\right)-G_{\text {memb }}(t, \cdot) \quad \text { in } \omega, \\
& 0=\operatorname{div} \operatorname{div}\left(\Sigma_{0}\left(\frac{1}{24} D^{2} v_{3}+\left[p^{1,2}\right]_{1}\right)\right)-g_{\text {bend }}(t, \cdot)-\operatorname{div} G_{\text {bend }}(t, \cdot) \text { in } \omega, \\
& 0 \in \partial \widetilde{R}^{0}(\mathbb{D} \dot{p})+\operatorname{dev} \llbracket \Sigma_{0}\left(p^{1,2}-\varepsilon^{1,2}(v)+x_{3} \mathrm{D}^{2} v_{3}\right) \| 0 \rrbracket+k_{\text {hard }} \mathbb{D} p \quad \text { in } \Omega,
\end{aligned}
$$

We see that (4.1a) is a second-order membrane equation for the in-plane displacements $v^{1,2}=\left(v_{1}, v_{2}\right)$ with the average plastic strains $\left[p^{1,2}\right]_{0}$ acting as plastic strains. The fourth-order equation (4.1b) for the out-of-plane displacement $v_{3}$ generalizes Kirchhoff's plate equation, where now the first moments $\left[p^{1,2}\right]_{1}$ (odd averages) act as plastic strains. The flow law (4.1c) is still posed on $\Omega=\omega \times(-1 / 2,1 / 2)$, but the important point is that the coupling with $\varepsilon^{1,2}(v)$ and $\mathrm{D}^{2} v_{3}$ occurs only via special $x_{3}$-dependent profiles, namely 1 and $x_{3}$, respectively.

\subsection{The choice of scalings for the plastic strains}

A careful inspection of the convergence proofs in Sect. 3 reveals that the results above remain valid for scalings of the form

$$
p_{h}\left(x_{h}\right)=\Pi_{h}^{\alpha, \beta} p^{h}\left(S_{h} x_{h}\right):=\left(\begin{array}{lll}
p_{11}^{h} & p_{12}^{h} & \frac{1}{h^{\alpha}} p_{13}^{h} \\
p_{12}^{h} & p_{22}^{h} & \frac{1}{h^{\alpha}} p_{23}^{h} \\
\frac{1}{h^{\alpha}} p_{13}^{h} & \frac{1}{h^{\alpha}} p_{23}^{h} & \frac{1}{h^{\beta}} p_{33}^{h}
\end{array}\right) .
$$

with $\alpha, \beta>0$. This means, scalings of this particular form lead to the same limit model. By replacing $\mathbb{D} p$ by $\tilde{p}$ in the previous section we see that for $\alpha, \beta>0$ the limit model is similar to the one derived in [11], where $\alpha=\beta=0$ was chosen. In [11] the components $p_{13}$ and $p_{23}$ of the plastic strains do not vanish, however, they stay constant since they are not triggered by the elastic stresses. Here, however, the strains $p_{13}$ and $p_{23}$ have to vanish due to the scalings. The component $p_{33}$ can be reintroduced due to the plastic incompressibility assumption.

\subsection{Prandtl-Ishlinskii operators}

The plastic flow rule for the limit system can be encoded in terms of vectorvalued Prandtl-Ishlinskii operators. We highlight here the main ideas and refer to [11] for a deeper discussion.

We note that (4.1c) can be written in the form

$$
0 \in \partial \widetilde{R}^{0}(\dot{\tilde{p}})+\mathbb{A} \widetilde{p}-\mathbb{L}(t)
$$

with the loading $\mathbb{L}=\operatorname{dev} \llbracket \Sigma_{0} \varepsilon^{1,2}(v)-x_{3} \mathrm{D}^{2} v_{3} \| 0 \rrbracket$ and $\widetilde{p}=\mathbb{D} p$. The vectorvalued play operator $\mathbb{P}$ maps the loading $\mathbb{L}$ to the solution $\widetilde{p}$, i.e.,

$$
\mathbb{P}_{K}[\mathbb{L}](t)=p(t),
$$

where $K=\partial \widetilde{R}^{0}(0)$. We now set

$$
\mathfrak{P}_{\text {memb }}\left[\varepsilon^{1,2}(v), \mathrm{D}^{2} v_{3}\right]=\left[\mathbb{P}_{K}[\mathbb{L}]\right]_{0}, \quad \text { and } \quad \mathfrak{P}_{\text {bend }}\left[\varepsilon^{1,2}(v), \mathrm{D}^{2} v_{3}\right]=\left[\mathbb{P}_{K}[\mathbb{L}]\right]_{1} \text {. }
$$


With these definitions, we can rewrite the system (4.1) as

$$
\begin{aligned}
\operatorname{div}\left(\Sigma_{0}\left(\varepsilon^{1,2}(v)-\mathfrak{P}_{\text {memb }}\left[\varepsilon^{1,2}(v), \mathrm{D}^{2} v_{3}\right]\right)\right) & =G_{\text {memb }}(t, \cdot), \\
\operatorname{div} \operatorname{div}\left(\Sigma_{0}\left(\frac{1}{24} \mathrm{D}^{2} v_{3}+\mathfrak{P}_{\text {bend }}\left[\varepsilon^{1,2}(v), \mathrm{D}^{2} v_{3}\right]\right)\right) & =g_{\text {bend }}(t, \cdot)+\operatorname{div} G_{\text {bend }}(t, \cdot) .
\end{aligned}
$$

\section{References}

[1] Abels, H., Mora, M.G., Müller, S.: The time-dependent von Kármán plate equation as a limit of 3d nonlinear elasticity. Calc. Var. Partial Differ. Equ. 41(1-2), 241-259 (2011). doi:10.1007/s00526-010-0360-0

[2] Attouch, H.: Variational convergence for functions and operators. Applicable Mathematics Series. Pitman, Boston (1984)

[3] Ciarlet, P.G., Destuynder, P.: A justification of the two-dimensional linear plate model. J. Mécanique 18(2), 315-344 (1979)

[4] Ciarlet, P.G.: Mathematical elasticity, Vol. II Theory of plates. Studies in Mathematics and its Applications, vol. 27. North-Holland Publishing Co., Amsterdam (1997)

[5] Dal Maso, G.: An introduction to $\Gamma$-convergence. In: Progress in Nonlinear Differential Equations and their Applications, vol. 8. Birkhäuser Boston Inc., Boston, MA (1993)

[6] Friesecke, G., James, R.D., Müller, S.: A hierarchy of plate models derived from nonlinear elasticity by Gamma-convergence. Arch. Ration. Mech. Anal. $\mathbf{1 8 0}(2), 183-236$ (2006)

[7] Guenther, R.B., Krejčí, P., Sprekels, J.: Small strain oscillations of an elastoplastic Kirchhoff plate. ZAMM Z. Angew. Math. Mech. 88(3), 199-217 (2008)

[8] Han, W., Reddy, B.D.: Plasticity. In: Interdisciplinary Applied Mathematics, vol. 9. Springer, New York (1999)

[9] Krejčí, P., Sprekels, J.: Elastic-ideally plastic beams and Prandtl-Ishlinskii hysteresis operators. Math. Methods Appl. Sci. 30(18), 2371-2393 (2007)

[10] Liero, M.: Herleitung eines elastoplastischen Plattenmodells mit Methoden der $\Gamma$-Konvergenz. Institut für Mathematik, Humboldt-Universität zu Berlin, Diplomarbeit (2008)

[11] Liero, M., Mielke, A.: An evolutionary elastoplastic plate model derived via Г-convergence. WIAS preprint no. 1583 (2010, to appear)

[12] Mielke, A.: Evolution in Rate-Independent Systems, Chapt. 6. In: Dafermos, C.M., Feireisl, E. (eds.) Handbook of Differential Equations, Evolutionary Equations, vol. 2, pp. 461-559. Elsevier B.V., Amsterdam (2005)

[13] Mielke, A., Roubíček, T., Stefanelli, U.: Г-limits and relaxations for rateindependent evolutionary problems. Calc. Var. Partial Diff. Equ. 31(3), $387-416$ (2008) 
[14] Mielke, A., Theil, F.: On rate-independent hysteresis models. NoDEA Nonlinear Diff. Equ. Appl. 11(2), 151-189 (2004)

[15] Millet, O., Cimetiere, A., Hamdouni, A.: An asymptotic elastic-plastic plate model for moderate displacements and strong strain hardening. Eur. J. Mech. A/Solids 22(3), 369-384 (2003)

[16] Morgenstern, D.: Herleitung der Plattentheorie aus der dreidimensionalen Elastizitätstheorie. Arch. Ration. Mech. Anal. 4, 145-152 (1959)

[17] Morgenstern, D.: Mathematische Begründung der Scheibentheorie (zweidimensionale Elastizitätstheorie). Arch. Ration. Mech. Anal. 3, 91-96 (1959)

[18] Reddy, B.D.: Existence of solutions to a quasistatic problem in elastoplasticity. In: Progress in partial differential equations: calculus of variations, applications (Pont-à-Mousson, 1991), Pitman Res. Notes Math. Ser., vol. 267, pp. 299-311. Longman Sci. Tech., Harlow (1992)

[19] Rockafellar, R.T.: Convex analysis. Princeton Landmarks in Mathematics, vol. xviii. Princeton University Press, Princeton (1997)

Matthias Liero

Weierstrass Institute for Applied Analysis and Stochastics

Mohrenstraße 39

10117 Berlin

Germany

e-mail: liero@wias-berlin.de

Thomas Roche

Department of Mathematics/M6

Technische Universität München

Boltzmannstraße 3

85747 Garching

Germany

e-mail: roche@ma.tum.de

Received: 23 March 2011.

Accepted: 15 September 2011. 